\title{
RDUS
}

Revue de DROIT UNIVERSITÉ DE SHERBROOKE

Titre : $\quad$ QUEL DROIT COMPARÉ?

Auteur(s): $\quad$ H. Patrick GLENN

Revue : $\quad$ RDUS, 2013, volume 43, numéro 1-2

Pages : $\quad 23-44$

ISSN : $\quad$ 0317-9656

Éditeur: $\quad$ Université de Sherbrooke. Faculté de droit.

URI : $\quad$ http://hdl.handle.net/1 $\quad$ 1143/10205

DOI : https://doi.org/10.17118/11143/10205 
Page vide laissée intentionnellement. 


\title{
ARTICLE
}

\section{QUEL DROIT COMPARÉ?}

\author{
par H. Patrick GLENN*
}

Il y a un choix à faire entre des types différents de droit comparé. Il y a d'abord le droit comparé qui a prévalu pendant les deux derniers siècles, un droit comparé dominé par le positivisme juridique et la logique dite classique, qui vise la description de systèmes juridiques conçus comme entités statiques. Il y a cependant un autre droit comparé, dominé par la comparaison normative et la logique paraconsistante. L'article examine d'abord l'état actuel du sujet pour ensuite se tourner vers le choix à faire entre ces types de droit comparé et les conséquences d'un tel choix.

There is a choice to be made between different types of comparative law. There is first of all the comparative law which has prevailed during the last two centuries, a comparative law dominated by legal positivism and so-called classical logic, which aimed at the description of legal systems conceived as static in character. There is, however, another comparative law, dominated by normative comparison and paraconsistent logic. The article examines the present state of the subject before turning to the choice to be made between these two types of comparative law and the consequences of such a choice.

Titulaire de la Chaire Peter M. Laing, Faculté de droit et Institut de droit comparé, Université McGill. 


\section{SOMMAIRE}

I. L'état actuel du sujet .................................... 26

II. Les options pour un droit comparé contemporain .......32

III. Les conséquences du choix d'une notion de droit comparé ............................................. 36

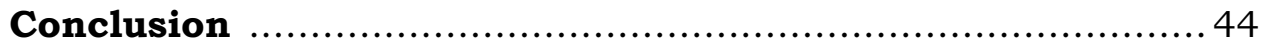


On parle souvent de la méthode comparative en droit, et l'expression est révélatrice de l'emprise de la pensée étatisée des deux derniers siècles. Charles Taylor nous a enseigné que la pensée normative ou pratique est par sa nature comparative ${ }^{1}$, mais l'étatisation du droit a gommé cette vérité en faveur de l'idée que la normativité juridique est locale et univocale. La comparaison des normes aurait donc son rôle à partir de la frontière, et à toutes fins utiles, mais nullement à l'intérieur des Pyrénées $^{2}$. Le droit comparé devient nécessairement, dans cet environnement intellectuel, une poursuite noble ouverte vers le monde, mais périphérique aux préoccupations juridiques principales. Il est à la remorque de l'idée dominante qu'il n'y a pas de place pour la comparaison dans le droit qui existe, isolé à l'intérieur de chaque État. Le titre de ces remarques est donc subversif, se joignant ainsi à d'autres propositions récentes ${ }^{3}$, en suggérant que le droit comparé ne doit pas nécessairement être compris dans ce sens, qu'il y a un choix à faire en parlant du droit comparé. C'est un choix qui doit être fait en fonction premièrement de l'état actuel du sujet (I), deuxièmement des options à considérer en effectuant ce choix (II) et troisièmement des conséquences prévisibles d'un tel choix (III).

1. Charles TAYLOR, Sources of the Self: The Making of the Modern Identity, Harvard University Press, 2009, p. 72 ("Practical reasoning ... is a reasoning in transition. It aims to establish, not that some position is correct absolutely, but rather that some position is superior to some other. It is concerned, covertly or openly, implicitly or explicitly, with comparative propositions").

2. Blaise PAscal, Les Pensées, Paris, Chez Lefèvre, 1826, p. 95 ("Plaisante justice qu'une rivière ou une montagne borde! Vérité au-deçà des Pyrénées, erreur au-delà!"). Il n'y aurait donc pas de découverte de la vérité, qui est déjà donnée à l'intérieur des Pyrénées, et, si la comparaison devient possible à la frontière, elle est essentiellement inutile, sauf aux fins de constatation.

3. Horatia MUIR WATT, "La fonction subversive du droit comparé ", R.I.D.C. 2000. 503; G. FletChER, "Comparative Law as a Subversive Discipline ", 46 Am. J. Comp. L. 683 (1988). 


\section{L'état actuel du sujet}

Selon des commentaires récents et autorisés, le droit comparé tire à sa fin, du moins comme discipline autonome et scientifique. Il y a plus d'une décennie, Mathias Reimann écrivait au sujet de la fin du droit comparé comme sujet autonome ${ }^{4}$ et, plus récemment, Mathias Siems l'a formulée comme la fin du droit comparé tout court ${ }^{5}$. Peer Zumbansen, pour sa part, a dit qu'après un siècle d'existence, le droit comparé " is not dead - or, is it? "6 et William Twining s'en moque gentiment pour sa "country and western tradition $"^{7}$. Annalise Riles trouve tout simplement que les grands projets comparatifs ont trop souvent désintégré "into a plethora of minutely detailed factual inquiries " 8 . Comment comprendre ces prises de position et leur pertinence pour l'état actuel du droit comparé?

Ces critiques de droit comparé se situent elles aussi dans un contexte intellectuel, et notamment à une époque où le rôle du droit étatique est mis sérieusement en question. On parle de plus en plus de droit transnational sinon plus généralement d'un processus de mondialisation, et avec raison ${ }^{9}$. La critique du droit comparé, un droit comparé qui regarde au-delà de l'État, a lieu en même temps que l'État lui-même est critiqué de plus en plus. Le

4. Mathias REIMANN, "The End of Comparative Law as an Autonomous Subject ", (1996) 11 Tul. Eur. \& Civ. L. F. 49; voir: M. REIMAnN, "The Progress and Failure of Comparative Law in the Second Half of the Twentieth Century ", (2002) 50 Am. J. Comp. L. 671.

5. Mathias SiEMS, "The End of Comparative Law ", (2007) 2 J. Comp. L. 133.

6. Peer Zumbansen, "Transnational Comparisons: Theory and Practice of Comparative Law as a Critique of Transnational Governance ", (2012) p. 2, en ligne : <http://ssrn.com/abstract=2000803>.

7. William Twining, General Jurisprudence, Cambridge, Cambridge Univ. Press, 2009, p. 10.

8. Annalise RILES, "Wigmore's Treasure Box: Comparative Law in the Era of Information ", (1999) 40 Harv. J. Int. L. 221, p. 239.

9. Voir H. Patrick GlenN, "A Transnational Concept of Law ", dans P. CANE et M. Tushnet, The Oxford Handbook of Legal Studies, Oxford, Oxford Univ. Press, 2003, à la p. 839; voir notamment Martin VAN CREVELD, The Rise and Decline of the State, Cambridge, Cambridge Univ. Press, 1999, p. 441. 
déclin de l'État ne semble pas impliquer un rôle accru pour le droit comparé. Il y aurait donc, paradoxalement, un lien étroit entre l'État moderne et le droit comparé. Les deux n'existeraient pas en opposition l'un à l'autre, comme visions différentes du monde juridique, mais plutôt en collaboration. La critique du droit comparé serait d'ailleurs une conséquence du déclin du droit étatique. Il faut chercher les origines de ce développement dans certaines idées qui entourent (et influencent) nos conceptions actuelles du droit.

Une première idée importante est celle du positivisme scientifique. Léontin-Jean Constantinesco a magistralement démontré les liens entre le droit comparé, tel qu'il a été conçu au dix-neuvième siècle, et des disciplines scientifiques comme l'anatomie comparée, la biologie comparée et la linguistique comparée ${ }^{10}$. Ces dernières ont joué un rôle de modèle pour le premier, comme d'ailleurs les sciences dures ont joué un rôle important dans le développement de l'idée de la science juridique. La science se caractériserait par ses méthodes en examinant les objets de la nature, et notre compréhension moderne de la comparaison est tirée directement de cette idée fondamentale d'objets d'étude. La comparaison, comme l'enseignent même les dictionnaires modernes, c'est la constatation de similarités et de différences entre les objets examinés ${ }^{11}$. C'est tout. Il n'y aurait pas de normativité dans le processus et les sciences comparatives seraient essentiellement des sciences descriptives ${ }^{12}$. Comparer les

10. Léontin-Jean Constantinesco, Traité de droit compare, t. 3, La science des droits comparés, Paris, Economica, 1983, p. 52-3, et pour le droit comparé p. 42-43.

11. Jean-Marie PRUVOst-BEAURAIN, Dictionnaire usuel illustré, Paris, Quillet/Flammarion, 1980 sous " comparer " ("Examiner les rapports de ressemblances existant entre des personnes, des choses "); Petit Larousse illustré, Paris, Larousse, 1980 sous " comparer " ("Examiner, établir les ressemblances ou les différences qui existent entre des personnes ou des objets "; Joyce M. Hawkins, The Oxford Reference Dictionary, Oxford, Clarendon Press, 1986 sous "compare" (" to estimate the similarity of one thing with or to another; two things").

12. Il y aurait cependant une normativité implicite ou cachée dans l'opposition de constatations ou de descriptions différentes. 
droits serait ainsi un processus de description ou de constatation de similarités et de différences des droits examinés. Si l'étudiant qui commence ses études en droit a l'idée que le droit est par sa nature normative, la notion de droit comparé paraît comme un oxymoron. En conséquence, les cours de droit comparé sont souvent boudés par les étudiants en droit. Ils sont périphériques aux préoccupations principales des étudiants, qui ont bien compris l'enseignement de la plupart de leurs professeurs.

Une deuxième idée importante est celle qui enseigne que la logique dite "classique " doit jouer un rôle important dans le droit. Cette logique classique a renforcé de manière importante le positivisme scientifique. Le positivisme scientifique enseigne qu'il faut étudier les objets de la nature; la logique classique enseigne, à travers sa " loi d'identité ", qu'il y a des objets à étudier. A est A, nous dit la loi d'identité, en écartant toute idée du caractère flou ou non statique de l'objet d'étude. Une fois acceptée la loi d'identité, d'ailleurs, suivent inexorablement les lois dites de la non-contradiction et du tiers (ou milieu) exclu ${ }^{13}$. On ne peut pas affirmer, logiquement, une proposition et sa contradiction (non [A et non-A]), et entre une proposition et sa contradiction il n'y a pas d'espace au milieu [A ou non-A]. L’importance de ces idées de la logique dans la construction des systèmes juridiques étatiques est incontestable. Kelsen a même enseigné que le système juridique moderne se caractérise par sa conformité avec la loi de la noncontradiction $^{14}$. Le mouvement de codification nationale avait

13. Le processus est inexorable une fois qu'une délinéation nette entre les objets ou les propositions est acceptée. Deux choses distinctes ne peuvent pas occuper le même espace, et entre une chose et sa contradiction le milieu est exclu car la contradiction commence là où la chose prend fin.

14. Hans Kelsen, La théorie pure du droit, Paris, Dalloz, 1962, p. 274 (" [...] la norme fondamentale assure l'unité de ces normes dans leur pluralité. Cette unité s'exprime aussi dans le fait qu'un ordre juridique peut être décrit en propositions de droit qui ne se contredisent pas ".) et à la p. 275 : "Tout de même qu'une contradiction logique, un conflit de normes représente une absurdité ". Plus tard dans ses écrits, cependant, Kelsen a admis la possibilité de normes à la fois contradictoires et valides; voir H. KELSEN, Essays in Legal and Moral Philosophy, choisis par 
comme objet principal l'élimination de contradictions ou d'antinomies à l'intérieur du droit étatique ${ }^{15}$.

La combinaison du positivisme juridique et de la logique classique a largement conditionné nos idées des systèmes juridiques nationaux, des relations entre eux, et du droit comparé. Un système, selon la théorie des systèmes, est un cadre à l'intérieur duquel les éléments du système sont en interaction. Selon le positivisme juridique, le système juridique peut exister et, selon la logique classique, il est autonome et indépendant. La France est la France (" une et indivisible " selon la Constitution de l'an I) ${ }^{16}$. Une fois chaque système juridique national ainsi reconnu, il s'ensuit qu'un seul d'eux est applicable sur un seul territoire (le principe de la territorialité), puisque on ne peut pas affirmer une chose et sa contradiction (le droit français et le droit non français). Le principe est appliqué même aux relations juridiques internationales, car le droit international privé insiste que chaque relation juridique privée doit être soumise à un seul des systèmes juridiques qui seraient en cause. En plus, il n'y aurait pas de milieu inclus entre deux droits étatiques, et seules les réponses des deux droits étatiques peuvent être prises en considération par le juge étatique. Le principe a exclu pendant très longtemps l'arbitrage, où l'arbitre est libre de chercher la solution particulière qui s'impose ${ }^{17}$. Les relations entre les droits étatiques sont ainsi conçues de manière conflictuelle, et la dite science du droit

O. Weinberger, trans. Heath, Dordrecht, D. Reidel, 1973, à la p. 235 (" that two mutually conflicting norms should both be valid, is possible").

15. Pour les principes de la non-contradiction et du tiers exclu comme fondements de la codification française, Denys DE BÉcHILLON, "L'imaginaire d'un Code" (1998) 27 Droits 173 à la p. 182; et pour l'absence en général de contradictions à l'intérieur des codifications nationales, Giacomo GavazzI, Delle antinomie, Turin, G. Giappichelli, 1959, p. 106.

16. Raoul Van Caenegem, An Historical Introduction to Western Constitutional Law, Cambridge, Cambridge Univ. Press, 1995, p. 187.

17. Au Québec, l'arbitrage a été vu comme contraire à l'ordre public jusqu'aux années soixante du siècle dernier; voir John E.C. BRIERLEY, "Quebec Arbitration Law : A New Era Begins ", (1985) 40 Arb. J. 31. 
international privé est surtout caractérisée, depuis Huber au dixseptième siècle, par la notion de conflit de lois ${ }^{18}$.

Le caractère descriptif du droit comparé a suivi les mêmes principes. Il y aurait un objectif scientifique simplement dans la description des systèmes juridiques nationaux, conçus comme entités statiques en conflit les unes avec les autres. En plus, l'objectif de description pourrait être complémenté par un objectif plus large et ambitieux, qui serait la catégorisation des systèmes juridiques selon leurs caractéristiques, définies en termes de familles de droit du monde. Le système juridique français appartiendrait à la famille romano-germanique, et ainsi de suite. En procédant ainsi à la catégorisation des systèmes juridiques, la science du droit comparé suivrait le modèle taxonomique des sciences de la nature, et il y aurait naturellement une discipline scientifique autonome de droit comparé et des spécialistes de cette discipline. En comprenant le droit comparé de cette manière les liens entre le droit étatique et le droit comparé deviennent plus évident. En les décrivant et en les catégorisant, les spécialistes de droit comparé renforcent l'existence et l'autonomie des systèmes juridiques nationaux. Ils occupent tout le champ du droit, et même le regard du comparatiste, qui dépasse un seul système juridique pour mieux catégoriser tous les systèmes juridiques, est

18. Au point où, en certains droits du continent européen, le conflit est présumé dans chaque situation internationale même en l'absence de toute allégation de différence entre les droits, de sorte que le juge doit appliquer d'office les règles du droit international privé du for, une solution heureusement évitée dans l'article 2809 C.c.Q. Sur les droits européens, T. C. HARTLEY, "Pleading and Proof of Foreign Law: The Major European Systems Compared", (1996) 45 I.C.L.Q. 271; et pour la position médiatrice du droit français actuel (règles du droit international privé applicable d'office seulement en cas de normes qui ne sont pas à la disposition des parties), Bénédicte FAUVARQUE-Cosson, Libre disponibilité des droits et conflits de lois, Paris, L.G.D.J., 1998. Pour Huber et son texte: Ernest G. LoREnZEN, "Huber's De Conflictu Legum ", dans Ernest G. Lorenzen, Selected Essays on the Conflict of Laws, New Haven, Yale Univ. Press, 1947, à la p. 136; et pour son œuvre comme un changement théorique complet, S. THORnE, "Sovereignty and the Conflict of Laws", dans S. E. THORNE, Essays in English Legal History, London, Hambledon Press, 1985, 171, à la p. 181. 
parfaitement compatible avec l'existence et l'autonomie de chacun d'eux. D'ailleurs, la pratique de la comparaison est limitée à un champ restreint de spécialistes. Le praticien du droit n'aurait pas à s'occuper des droits étrangers. Dans certains pays, d'ailleurs, cette possibilité serait positivement exclue en insistant sur l'application d'office par le juge des règles de droit international privé et l'envoi du dossier régi par une loi étrangère à un institut universitaire pour la détermination de sa résolution selon le droit étranger ${ }^{19}$.

Le droit comparé sort de ce processus bien sûr comme une discipline noble, digne $\mathrm{du}$ nom de science, mais terriblement limité dans ses fonctions et dans ses ambitions. Même défini de cette manière, d'ailleurs, le projet taxonomique du droit comparé a donné lieu à un échec retentissant ${ }^{20}$. Parmi les comparatistes du dix-neuvième et du vingtième siècles, il n'y avait pas d'accord sur la définition des familles de droit, et le débat sur cette question a largement épuisé les participants. Il y avait peu ou pas de taxonomie effectivement entreprise. Si les auteurs cités au début de cette section préconisent la fin du droit comparé, c'est donc en visant la discipline telle qu'elle a été définie pendant la plupart de son existence moderne. Il reste la question de savoir si le droit comparé peut être conçu d'une autre manière, et si une notion plus dynamique du droit comparé est compatible avec les circonstances actuelles du monde.

19. Supra, note 18 .

20. Voir H. Patrick Glenn, "Comparative Legal Families and Comparative Legal Traditions", dans Mathias Reimann et Reinhard Zimmermann, The Oxford Handbook of Comparative Law, Oxford, Oxford Univ. Press, 2006, 421 notamment aux p. 424-425, et voir aussi aux p. 434-455 pour le caractère eurocentrique de la comparaison qui porte uniquement sur les États et leurs systèmes juridiques nationaux, axée ainsi presque exclusivement sur des sources de droit d'origine européenne. Pour la même critique en ce qui concerne la Chine contemporaine et la place des normes du "Sinomarxismus ", H. VON SENGER, "Von der Vergleichung des Rechts zur Vergleichung der Gesellschaftsfûhrung " (2006) ZfRV 43, notamment à la p. 55, citant Rheinstein. 


\section{Les options pour un droit comparé contemporain}

Une première option pour un droit comparé contemporain est l'option que nous venons d'examiner, qui reste bien vivante, notamment sous forme du Traité en trois volume du professeur Constantinesco, le troisième volume ayant été publié en $1983^{21}$. L'option est bien vivante aussi dans le caractère répandu de ses concepts et de son langage. Le langage de systèmes juridiques, de familles de droit et de sciences juridiques est aujourd'hui incontournable. Ce langage, il est vrai, n'est pas parfaitement compatible avec les phénomènes contemporains de droit transnational, de "dialogue judiciaire" et d'un droit dit "cosmopolite". Nous ne pouvons pas, cependant, abolir les concepts et les mots, et ces concepts et ces mots auront une réception différente dans différents endroits du monde. Il faut donc vivre avec des options différentes de droit comparé. L'essentiel, c'est qu'il y a des options, sans que cela implique une frontière nette, à tous les égards, entre les options. Si la comparaison des droits peut viser et accommoder des droits très différents, elle est suffisamment large pour accommoder des notions différentes de droit comparé. Les gens qui font la comparaison des droits partagent certains objectifs, sinon tous les objectifs, de la comparaison.

Une autre option pour le droit comparé est nécessairement incomplète, parce qu'elle n'a pas été l'objet de développements poussés et récents. Puisque le droit n'existe pas en vase clos, cependant, il est possible de chercher des fondements d'une autre option dans les mêmes disciplines qui ont largement contribué à notre première option. Si ces fondements ont été ébranlés ailleurs et ont cédé à d'autres idées ou fondements, ces autres idées ou fondements devraient être intéressants eux aussi pour le droit comparé.

Où en est-on avec le positivisme scientifique et son processus taxonomique aujourd'hui? Les choses ont évolué depuis

21. Supra, note 10. 
le dix-neuvième siècle. Si on ne peut être que schématique, une conclusion s'impose. Il y a moins de certitude aujourd'hui quant à l'existence des choses, et moins de certitude quant à leur catégorisation. Il y a moins de certitude quant à l'existence des choses parce que la physique contemporaine a mis en question la notion de masse et ses éléments constitutifs. Il y aurait un monde de l'infiniment petit, ce qui veut dire que nous ne pouvons plus séparer clairement les objets de l'examen des instruments de l'examen. Le proton ne peut plus être considéré simplement comme une boule en miniature mais comme un phénomène changeant et contradictoire 22 . Le travail de l'accélérateur géant de CERN à Genève a été construit à grands frais pour la recherche de l'infiniment petit. On ne connaît pas encore les résultats, et on ne connaît surtout pas leurs conséquences.

C'est aussi le cas, et on peut dire nécessairement, que la taxonomie biologique ne peut plus être ce qu'elle ne l'était. Si la taxonomie du dix-neuvième et du vingtième siècles rangeait les choses dans leur place dans le monde, la taxonomie du vingt-etunième siècle est plus circonspecte. Les espèces aujourd'hui ne peuvent plus être vues comme des classes statiques ou simplement comme des organismes. Elles représentent plutôt des lignées en évolution (" evolving lineages ") et leur catégorisation est variable, impliquant souvent le "cluster analysis " (le groupement de caractéristiques similaires dont nulle n'est définitive) ou la situation par rapport à une communauté de descendance ${ }^{23}$. Il y a donc un défi ontologique, en biologie aussi bien qu'en physique, au positivisme scientifique. Ce défi a nécessairement des conséquences pour les constructions intellectuelles, telles que le droit, qui se sont appuyées de façon métaphorique sur l'existence matérielle des choses. La métaphore n'a plus la même force.

22. Frank WILCZEK, The Lightness of Being: Mass, Ether and the Unification of Forces, 2010, p. 43 et 54. M. Wilczek est un lauréat du Prix Nobel en matière de physique.

23. Marc ERESHEFSKY, The poverty of the Linnaean hierarchy: a philosophical study of biological taxonomy, Cambridge, Cambridge Univ. Press, 2001, aux p. 3 (pour les lignées en évolution) et 15 (pour le "cluster analysis " et les communautés de descendance). 
Le défi ontologique a des conséquences aussi pour la logique "classique " et ses méthodes de travailler avec des concepts nets et éternels ("A ", "non-A ", etc.). Notamment la loi d'identité (A est A) a l'air remarquablement naïf face aux enseignements de la science moderne. $Y$ a-t-il quelque chose d'aussi simple et net que " $\mathrm{A}$ " dans le monde, et sinon comment la logique classique peut-elle trouver application dans un monde complexe et même évolutif? Plus particulièrement, ladite loi de la non-contradiction et la loi du tiers exclu, qui ont tellement influencé la construction des systèmes juridiques contemporains, peuvent-elles garder leur validité si les frontières des "A " du monde n'ont plus la même fixité, en devenant floues et perméables? 24 On commence à avoir des réponses à ces questions, des réponses des " nouvelles logiques " qui mettent en question la logique dite classique.

Ce que les "nouvelles logiques " cherchent surtout à faire, c'est de fournir une manière de penser qui serait adéquate pour un monde en mouvement et plein de contradictions. Ces nouvelles logiques sont donc "paraconsistantes " ou "polyvalentes " en acceptant qu'il n'est pas illogique de vivre avec une ou des contradictions. On peut vivre, et rendre jugement, dans un nouveau milieu inclus, défini à ces extrémités par les éléments contradictoires. La démonstration est faite même avec les concepts nets de la logique classique, en insistant sur les paradoxes récursifs ("Cette phrase est fausse") qui sont à la fois vrais et faux ${ }^{25}$. Le choix univalent de leur caractère vrai ou faux ne s’impose pas. La loi du tiers ou du milieu exclu ne tient pas. Si la

24. Voir George KlIR, U. St. Clair et Bo YuAn, Fuzzy Set Theory: Foundations and Applications, Upper Saddle River NJ, Prentice Hall, 1997, p. 75 ("Moreover, if we allow objects, and sets generally, to have imprecise boundaries, then the two classically important principles - the laws of contradiction and excluded middle - will no longer always be true ").

25. Voir notamment Graham PRIEST, In Contradiction [:] A Study of the Transconsistent, Oxford, Clarendon Press, 2006, ch. 1 et 2, rejetant aux p. 5 et 6 ledit principe de l'explosion (ex contradictione quodlibet) à l'effet que n'importe quelle conclusion découle d'une contradiction, pour les motifs que toutes les conclusions ne sont pas vraies et que "inconsistency does not entail incoherence". 
démonstration peut ainsi être faite dans le monde formel et austère de la logique classique, il est d'autant plus évident qu'il peut être fait dans le monde informel de tous les jours, et notamment dans le monde du droit. Le droit au respect de la vie privée et la liberté d'expression sont évidemment contradictoires, mais on peut affirmer les deux en même temps (la loi de la noncontradiction ne tient pas) et la jurisprudence sur la question répond aux deux valeurs en situant chaque décision à un point précis dans le milieu inclus.

On dit que le défi des nouvelles logiques à la logique classique a déjà réussi26. Il y aurait donc actuellement un " multi valued turn " dans la logique 27 . Le mouvement date du début du vingtième siècle, mais s'est accéléré dans la deuxième moitié du siècle ${ }^{28}$. Il a été propulsé notamment par les critiques sévères de Stephen Toulmin à l'égard de la logique classique, qui a contrasté sa stérilité avec la logique plus souple et complexe... du droit 29 . Les juristes auraient donc toujours fait de la logique nouvelle, de la même manière que $M$. Jourdain faisait la prose. Ce qui semble fondamental dans la situation actuelle, c'est qu'il y a des logiques dans le monde et un choix de logique doit être fait dans chaque

26. Katalin Bimbò "Relevance Logics ", dans Dale JacQuetTe (dir.), Philosophy of Logic, Amsterdam, Elsevier, 2007, à la p. 723 (" once the overpowering dominance of classical logic has been successfully challenged (and it has been) ").

27. Dov GabBay et John Woods. Jr., The Many Valued and Nonmonotonic Turn in Logic, Amsterdam, North-Holland, 2007.

28. Siegfried GotTEWALD, "Many-Valued Logics" dans Dale JAcQuetTe, Philosophy of Logic, Amsterdam, Elsevier B.V., 2007, p. 675-680 (citant Lukasiewicz et Post dans les années vingt) et p. 681 (" préhistoire " des situations futures d'Aristote); Grzegorz MALINOwSKI, "Many-valued Logic ", dans GaBbay et Woods; The Many Valued and Nonmonotonic Turn in Logic, à la p. 14 (pour la bataille marine d'Aristote comme fondement des logiques polyvalentes)

29. Stephen E. Toulmin, The Uses of Argument, Cambridge, Cambridge Univ. Press, 1958, aux p. 7 ("Logic (we may say) is generalized jurisprudence "), 39 (posant la question "How far is a general logic possible? ") et 147 (logiciens ayant une notion restreinte de la validité des arguments, cherchant à juger des arguments en termes qui sont "field invariant"). 
situation de réflexion et de jugement. Il y aurait un pluralisme logique actuel ${ }^{30}$. D'ailleurs, puisqu'il y a maintenant une logique polyvalente, le choix entre elle et la logique classique ne peut pas être un choix dicté par les méthodes de la logique classique. Seule une logique polyvalente de deuxième ordre permet l'affirmation des deux types de logique, d'un milieu inclus entre eux, et de la possibilité de choisir l'une ou l'autre dans des situations précises. On assiste déjà à un débat portant sur les vertus des deux types de logiques pour des domaines précis du droit ${ }^{31}$. La logique du droit fiscal ne doit pas être la logique du droit constitutionnel et des libertés publiques.

Dans le choix d'une notion de droit comparé, on peut donc vivre (comme il a été indiqué précédemment) avec une notion taxonomique et étatisée du droit comparé en parallèle avec une notion plus dynamique, plus polyvalente, et plus engagée du droit comparé. Il reste à déterminer les conséquences de ce choix de logique et de droit comparé dans différents domaines de droit.

\section{Les conséquences du choix d'une notion de droit comparé}

On connaît assez bien les conséquences du choix de droit comparé qui prévalait au dix-neuvième et au vingtième siècles. La notion de comparaison était fort restreinte; sa pratique était limitée aux spécialistes de la comparaison; les relations entre les systèmes juridiques étaient limitées et conflictuelles. Cette notion reste avec nous mais devient de plus en plus dépassée par la délocalisation du droit ${ }^{32}$. Que peut-on prévoir comme consé-

30. J.C. Beal et G. Restall, Logical Pluralism, Oxford, Oxford Univ. Press, 2006.

31. Voir Timothy A.O. EndicotT, Vagueness in Law, Oxford, Oxford Univ. Press, 2000, p. 73 (au moins pour les causes de validité/invalidité, culpabilité/innocence " graded standards might be undesirable").

32. Beck et Lau parlent peut-être plus précisément d'enlèvement des frontières ou "Entgrenzung "; voir U. BECK et C. LAU, Entgrenzung und Entscheidung: Was is neu an der Theorie reflexiver Modernisierung?, Frankfurt, Suhrkamp, 2004. 
quences du choix d'une notion polyvalente ou dynamique du droit comparé?

La conséquence principale d'un tel choix est l'ouverture vers d'autres droits et d'autres valeurs que ces droits incarnent. C'est l'engagement qui caractérise un droit comparé polyvalent, et non plus la séparation et l'exclusion. Le champ des droits reconnaissables est donc élargi, aussi bien que leurs champs possibles d'application, et la participation dans le monde de droit comparé devient plus inclusive et ouverte. On peut pratiquer le droit comparé en s'occupant, comme praticien, de l'application possible de plusieurs droits. Les occasions pour le faire se multiplient, dans tous les domaines du droit.

La place pour un droit comparé polyvalent est évidente dans le droit qui est connu comme international, et qui s'occupe des relations des systèmes juridiques nationaux du monde. Pendant deux siècles le droit international privé a été marqué par l'idée que les relations de droit privé peuvent être géographiquement localisées, selon des règles formelles de localisation. La logique, comme nous l'avons vu, est classique; un seul système juridique est applicable à chaque relation, le cumul de deux lois nationales est impossible. Il n'y a pas de comparaison, en principe, des droits quant à leur contenu, simplement un processus de localisation. Les changements dans la logique du droit international privé sont pourtant nombreux aujourd'hui et tous les changements comportent une notion de comparaison de droits qui est polyvalente en caractère. Cinq exemples peuvent être cités. Il y a d'abord la pratique de l'arbitrage, qui permet à l'arbitre de formuler une solution au litige qui n'est pas nécessairement la solution de l'un des systèmes juridiques nationaux en présence. Il peut comparer ces solutions en créant sa solution. Les parties à l'arbitrage sont aussi libres de choisir l'application des principes juridiques de deux systèmes juridiques nationaux, ce qui implique un processus actif de 
comparaison ${ }^{33}$. Ensuite, il y a le rôle de l'ordre public dans la résolution des causes de droit international privé. En principe l'ordre public joue un rôle très restreint, dans la formulation classique, et se limite simplement à écarter un droit étranger radicalement contradictoire avec le droit du for. La comparaison des droits a lieu, mais est soumise à la nécessité d'un choix unique. Récemment cependant, la Cour de cassation en France a admis que l'ordre public peut être un moyen de conciliation de lois fort différentes quant à leur contenu ${ }^{34}$. Le divorce islamique qui serait contraire à l'ordre public du for, pour sa violation du principe de l'égalité des sexes, peut être reconnu en France si le mari a fourni une protection pécuniaire adéquate à la femme. On évalue les résultats de l'application des droits. Troisièmement, une tendance marquée consiste à choisir la loi applicable à une cause internationale en fonction du résultat visé. Le professeur Symeonides a constaté le phénomène de "result selectivism " sur le plan mondial ${ }^{35}$; dans le Code civil du Québec, nous avons les articles 3091 (filiation), 3099 (successions), 3109 (forme des actes juridiques), 3117 (contrat de consommation), 3118 (contrat de travail) et 3128 (responsabilité civile) qui dictent tous l'application d'une loi, non pas en fonction de rattachements géographiques, mais en fonction de l'aptitude des lois en présence à fournir un résultat souhaité par le législateur. De plus, il y a la notion de l'" équivalence " des droits nationaux, selon laquelle il ne faut pas procéder au choix d'une seule loi applicable si les deux lois nationales sont équivalentes sur le fond. On arrive à cette conclusion par la comparaison, qui remplace la localisation. La notion est maintenant acceptée par la Cour de cassation en

33. Pour le choix de principes juridiques communs à la France et à l'Angleterre pour résoudre les différends dans la construction du Chunnel : C. C. SchÜTZ, "The Effects of General Principles of Law ", dans Dennis CAMPBEll, International Dispute Resolution, Alphen aan den Rijn, The Netherlands, Kluwer Law International, 2010, p. 43-45.

34. Guy CANIVET, "La convergence des systèmes juridiques du point de vue du droit privé français ", R.I.D.C. 2003.7.16-22 ("La fonction médiatrice de l'ordre public ").

35. S. SymeOnides, "Result Selectivism in Conflicts Law " 46 Willamette L. Rev. 1 (2009). 
France ${ }^{36}$. Finalement, existe la possibilité pour les parties de choisir une loi autre qu'une loi étatique et de voir ce choix respecté, au moins par les arbitres et possiblement par un juge étatique $^{37}$. Passer au-delà des sources étatiques à des sources non étatiques implique nécessairement un processus élargi de comparaison des droits.

Le droit international public est remarquable, comme le droit international privé, pour sa logique classique et exclusiviste. Seuls les États sont sujets de droit international public; en principe, le contenu de leurs lois internes n'entre pas en ligne de compte; le droit international public constituerait un bloc de normes, universelles en principe, qui ne comporte pas la nécessité de comparaison. On peut discerner l'idée d'un système de droit international qui partagerait des caractéristiques des systèmes nationaux: ils seraient tous les deux univoques dans leur articulation du droit. De nos jours, cependant, le langage du droit international public change; la comparaison entre dans ce droit, au point où on peut voir un changement même dans la nature de la discipline. D'abord, le droit international public n'est plus limité aux "pays civilisés ", comme il l'était pendant une bonne partie des deux derniers siècles ${ }^{38}$. Le droit international public des pays

36. Voir, pour la jurisprudence, S. GODECHOT-PATRIS, "Retour sur la notion d'équivalence au service de la coordination des systèmes ", Rev. crit. d.i.p. 2010.271-273 (qualifiant correctement la notion comme " un recul de la méthode conflictuelle").

37. L'option est écartée pour le juge étatique par Rome I (malgré la position de la Commission européenne), mais toujours envisagée par le Groupe de Travail de la Conférence de La Haye sur les contrats internationaux; voir Nils W. VernoolJ, "Rome I: An Update on the Law Applicable to Contractual Obligations in Europe" 15 Col. J. Eur. L.F 71, note 20 (2009), en ligne à <http://www.cjel.net>; pour les travaux à La Haye : $\mathrm{HccH}$, Draft Hague Principles on Choice of Law in International Contracts, 12-16 November 2012, en ligne : <http://www.hcch.net>.

38. Pour l'idée, et sa perte d'influence : Yasuaki OnUMA, "When was the Law of International Society Born? - An Inquiry of the History of International Law from an Intercivilizational Perspective ", (2000) 2 J. Hist. of Int'l L.1, p. 26; Bret BowDEN, "The Colonial Origins of International Law. European Expansion and the Classical Standards of Civilization ", (2005) 
occidentaux fait donc face à d'autres idées de communauté, sur le plan international. Il existe notamment l'umma, la communauté islamique, et des notions de loyauté personnelle qui n'ont jamais été écartées pleinement par la notion de l'État dans la pensée hindoue. Ces autres moyens de penser les communautés internationales se font sentir de manière inéluctable dans les négociations internationales et le comportement des États. Les internationalistes, et les diplomates, ont maintenant pour tâche de comprendre la perspective des autres, ce qui ne figurait pas dans la compréhension du droit international public durant la période active de sa construction. Le droit international public est cependant aujourd'hui fleurissant, du moins à l'égard de la multiplication de ses instances. Il existe de plus en plus de fors internationaux, de plus en plus de sources de droit international. On parle aussi donc de la "fragmentation " du droit international public, dans le sens qu'il n'y a plus de corpus uniforme de règles mais un vaste théâtre juridique, vulnérable à des influences venant de partout ${ }^{39}$. Il faut donc comparer les sources du droit international public, à l'intérieur de ce droit, dans sa nouvelle conception large. La notion de "droit international comparé " est ainsi née ${ }^{40}$. Inévitablement, dans son engagement avec d'autres notions de communautés humaines, et d'autres sources, le droit international public change dans son contenu. Il est affecté par la comparaison dynamique et polyvalente. Le résultat, dans le langage de Thomas Meron, c'est que le droit international public "s'humanise" en acceptant que la personne humaine devienne sujet et objet du droit international public ${ }^{41}$. Trois exemples viennent immédiatement à l'esprit: l'émergence d'un droit international des droits de la personne (avec des cours de justice

7 J. Hist. of Int'l L.1; S. J. ANAYA, Indigenous Peoples in International Law, 2e éd., Oxford, Oxford Univ. Press, 2004, p. 27-31.

39. Pour la fragmentation, Martti Koskenniemi et Päivi LEINO, "Fragmentation of International Law? Postmodern Anxieties ",(2002) 15 Leiden J. Int. L. 553, 559 (" a kaleidoscope reality ").

40. Martti Koskenniemi, "The Case for Comparative International Law ", (2009) 20 Finnish Yearbook of Int. L.3.

41. Theodor MERON, The Humanization of International Law, The Hague, Martinus Nijhoff, 2006. 
pour assurer son respect) ${ }^{42}$; l'émergence d'un droit pénal international pour sanctionner notamment les crimes contre l'humanité 43 ; et la reconnaissance des peuples indigènes (et leur droit non écrit) comme participants dans la création du nouveau droit international public ${ }^{44}$. Dans les trois cas, le droit en cause est nécessairement comparatif dans sa formulation, puisque le droit international public manquait un droit de fond applicable aux individus. Le nouveau droit doit nécessairement venir de l'enseignement des droits actuels, de manière comparative. Ainsi la jurisprudence sur les droits de l'homme circule de manière transnationale; le droit pénal international (tout comme la procédure pénale internationale) tire ses sources de multiples traditions juridiques; et les peuples indigènes participent activement dans les fors internationaux à partir des principes de leur droit non écrit. Le droit international public, comme le droit international privé, s'approche du droit comparé, qui lui doit s'occuper de vrais problèmes et non pas simplement de problèmes taxonomiques.

Si le droit international, privé et public, intègre de plus en plus la comparaison normative, le lien nécessaire avec une logique polyvalente n'est pas passé inaperçu. Ainsi, Mireille Delmas-Marty fait appel à la pleine "palette " de la logique moderne en ordonnant la multiplicité des droits du monde ${ }^{45}$ et François Rigaux a écrit de "l'illusion " de la catégorisation par le moyen des dichotomies et de la "perversité " de taxonomies binaires ${ }^{46}$; Christine Bell, dans son Lex Pacificatoria, écrit de la nécessité

42. Notamment la Cour européenne des droits de l'homme et la Corte interaméricana de derechos humanos.

43. Voir Antonion CASSESE, International Criminal Law, 2e éd., Oxford, Oxford Univ. Press, 2008.

44. H. Patrick GLenN, "The Three Ironies of the UN Declaration on the Rights of Indigenous Peoples ", dans Stephen ALLEN et Alexandra XANTHAKI, Reflections on the UN Declaration on the Rights of Indigenous Peoples, Oxford, Hart, 2011, p. 171.

45. Mireille DELMAS-MARTY, Les forces imaginantes du droit; Le relatif et l'universel, t. I, Paris, Seuil, 2004, p. 412.

46. François RigauX, La loi des juges, Paris, Odile Jacob, 1997, p. 69 et 250251. 
d'aller au-delà des distinctions binaires pour développer une " ambiguïté constructive" dans les relations entre des peuples ${ }^{47}$. De manière générale, dans le monde de la common law, Martin Krygier se fait critique des dichotomies "pernicieuses " qui "pourraient être simplement des aspects de phénomènes complexes capables d'inclure les deux "48. Au niveau de la Cour internationale de justice, dans le jugement récent portant sur la déclaration d'indépendance de Kosovo ${ }^{49}$, le juge Simma, tout en partageant l'avis de la majorité de la Cour, a mis en question tout le processus de raisonnement juridique binaire de cette majorité. Pour le juge Simma, l'absence d'une prohibition explicite d'une telle déclaration n'implique pas nécessairement son autorisation. Il a critiqué ce raisonnement binaire comme " obsolète " et a prôné une réponse plus nuancée et compréhensive qui permettrait une gamme de réponses, incluant ce qui serait "toléré ", " permissible " et "souhaitable "50. Ainsi les nouvelles logiques entrent dans le raisonnement juridique et judiciaire, comme éléments indispensables d'une comparaison active des droits.

Ce qui est vrai au niveau international se révèle aussi être le cas sur le plan du droit interne, même au point que la dichotomie entre l'interne et l'international s'affaiblit. C'est le dit " dialogue des juges" qui représente son développement le plus significatif. S’il y avait toujours une pratique de citation de jugements étrangers à l'intérieur du monde de la common law, le processus est maintenant plus répandu et est accompagné de communications judiciaires plus intenses et plus... comparatives. Les juges s'organisent des colloques, des réunions annuelles, et

47. Christine Bell, On the Law of Peace: Peace Agreements and the Lex Pacificatoria, Oxford, Oxford Univ. Press, 2008, p. 166, et voir aussi p. 302 (acceptant ce qui serait autrement un milieu exclu).

48. M. KRYGIER, "False Dichotomies, True Perplexities, and the Rule of Law ", dans András SAJó, Human Rights with Modesty: The Problem of Universalism, Leiden, Martinus Nijhoff, 2004, p. 251.

49. Conformité au droit international de la déclaration unilatérale d'indépendance relative au Kosovo, avis consultatif, (22 juillet 2010), C.I.J. Recueil 2010 , p. 403 (Cour internationale de Justice), en ligne : $<$ http://www.icj-cij.org>.

50. <Http://www.icj-cij.org/docket/files/141/15993.pdf>, aux par. 2 et 3. 
même des organisations transnationales 51 . Le processus traverse non pas seulement les frontières nationales, mais aussi le summa divisio entre le droit privé et le droit public. Si le droit privé a toujours connu une certaine ouverture, le droit public a résisté plus vigoureusement, et Otto Kahn Freund a écrit au vingtième siècle que la comparaison en droit public constituait même un "abus " de droit comparé52. Aujourd'hui le droit constitutionnel comparé est devenu un champ actif de comparaison, et au niveau doctrinal et au niveau judiciaire ${ }^{53}$. Au niveau de la pratique du droit, d'ailleurs, les professions juridiques ne sont plus cantonnées par les juges, les institutions universitaires et les frontières nationales dans leur appréciation des droits étrangers. L'arrivée de grands cabinets juridiques a fait en sorte que la comparaison des droits est pratiquée régulièrement à l'intérieur de ces cabinets, qui jouent un rôle important dans les choix d'un droit applicable et même, devant des arbitres, dans le choix d'une solution transnationale pour des causes transnationales ${ }^{54}$. Ainsi, tous les acteurs du monde du droit voient la possibilité de faire le droit comparé, de manière active et polyvalente, s'ouvrir devant eux.

51. Guy CAnivet, "Trans-Judicial Dialogue in a Global World " dans Sam Muller et Sidney Richards, Highest Courts and Globalisation, The Hague, Hague Academic Press, 2010, 21, notamment p. 24 (sites web portant sur la collaboration judiciaire), 31 (sur le développement de la "diplomatie judiciaire") et 34 (programmes d'échanges judiciaires, réseaux de juges).

52. Otto KAHN-FrEUnd, "On Uses and Misuses of Comparative Law ", (1974) 37 Mod. L. Rev. 1.

53. Pour la doctrine, voir notamment: Michel Rosenfeld et András SAJO, Oxford Handbook of Comparative Constitutional Law, Oxford, Oxford Univ. Press, 2012; et pour la pratique (établie mais controversée) de la citation des causes étrangères dans les décisions constitutionnelles de la Cour suprême des États-Unis: Graham v. Florida, 130 S. Ct. 2011 (2010), section D de l'avis de la Cour, où le juge Kennedy, pour la majorité, cite du droit étranger et la Convention internationale relative aux droits de l'enfant, non ratifiée aux États-Unis.

54. H. Patrick GlenN, "Comparative Law and Legal Practice: On Removing the Borders ", (2001) 75 Tulane Law Review 977. 


\section{Conclusion}

Faut-il avoir peur de la comparaison des droits? Un monde où les sources de droit se multiplient et s'entrecroisent est-il un monde d'incertitude et de conflit? Il faut se rappeler que la notion de conflits de lois est d'origine récente et s'est développée en même temps que la construction des droits étatiques. Avant le 17 e siècle, il n'y avait pas de conflits de lois, seulement des domaines de droit, de sources différentes, applicables sur un même territoire. C'était une époque de conciliation, et non pas de conflits, de lois. La conciliation s'effectuait par un processus, actif, de comparaison. La comparaison des droits devient ainsi un moyen pour faciliter la coexistence, la convivencia, des peuples. 